Images Re-vues

re-VuES

Histoire, anthropologie et théorie de l'art

Objets mis en signe

\title{
Objets flottants
}

Mette Kia Krabbe Meyer

\section{(2) OpenEdition}

\section{Journals}

Édition électronique

URL : http://journals.openedition.org/imagesrevues/119

DOI : 10.4000/imagesrevues. 119

ISSN : 1778-3801

\section{Éditeur :}

Centre d'Histoire et Théorie des Arts, Groupe d'Anthropologie Historique de l'Occident Médiéval, Laboratoire d'Anthropologie Sociale, UMR 8210 Anthropologie et Histoire des Mondes Antiques

\section{Référence électronique}

Mette Kia Krabbe Meyer, « Objets flottants », Images Re-vues [En ligne], 4 | 2007, document 2, mis en ligne le 01 janvier 2007, consulté le 30 janvier 2021. URL : http://journals.openedition.org/ imagesrevues/119; DOI : https://doi.org/10.4000/imagesrevues.119

Ce document a été généré automatiquement le 30 janvier 2021.

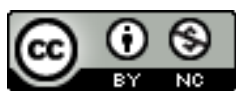

Images Re-vues est mise à disposition selon les termes de la Licence Creative Commons Attribution Pas d'Utilisation Commerciale 4.0 International. 


\title{
Objets flottants
}

\author{
Mette Kia Krabbe Meyer
}

1 Quels rapports pourrait-on imaginer entre des photos relevant de la publicité, du photomontage surréaliste et de l'occultisme? Dans le cas de la photographie danoise de l'entre-deuxguerres, dont il sera ici question, les ressemblances entre ces trois domaines photographiques s'avèrent frappantes. Une transformation de l'objet, qui semble lui donner vie, apparaît en effet dans chacun de ces trois domaines.

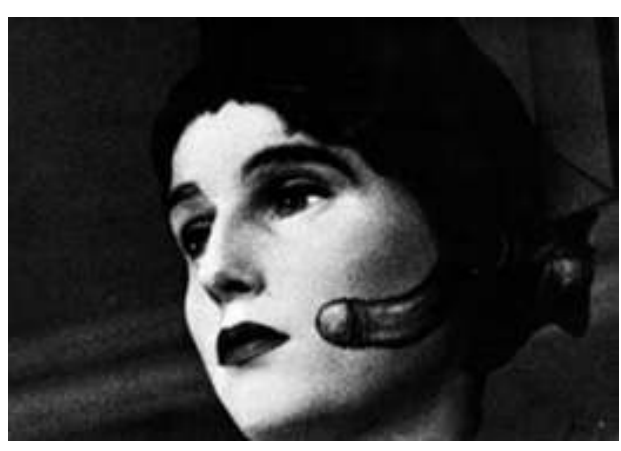

2 À cette époque, les photographes publicitaires commençaient à mettre en scène les produits d'une manière de plus en plus attrayante. Dans les milieux artistiques, on avait également commencé à s'intéresser à la nature des objets, et à photographier à la fois les objets commerciaux et les objets ethnographiques ou naturels. Enfin, les spirites photographiaient des objets comme les tables ou pendules utilisées dans leurs séances.

3 L'animation des objets inanimés avait été traitée, comme on le sait, par Sigmund Freud dans l'article "Das Unheimliche"paru en 1919 et où il évoque le concept d' « inquiétante étrangeté ${ }^{1}$. Freud parlait entre autres choses de poupées de cire et d'automates à figure humaine qui paraissaient étrangement vivants. Cependant Freud avait une approche psychanalytique, à partir de laquelle il a formé sa propre notion restreinte d'«inquiétante étrangeté ». Je ne suivrai pas la ligne psychanalytique de Freud dans cet article. D'un point de vue théorique, je me réfèrerai au médecin allemand Ernst Jentsch, qui a inspiré Freud, et à sa définition plus générale de «Das Unheimliche $»^{2}$. Dans la photographie danoise de l'entre-deux-guerres, on trouve un grand nombre d'objets divers qui semblent avoir leur propre vie. Et à la manière de Freud et de Jentsch, j'ajouterai aux objets animés, les cadavres. Le docteur Jentsch donnait comme exemple d'« inquiétante étrangeté » le corps du malade mental, qui se crispe de convulsions obsessionnelles, et Freud parlait de la notion de double, ce qui, 
pour lui, mettait en doute l'existence unique et donc l'existence « organique » de l'être humain.

4 Ici ce sont d'autres "corps mécaniques", qui m'intéressent: premièrement le mannequin, deuxièmement le corps dans les œuvres surréalistes et, troisièmement, les médiums spirites.

\section{Les objets animés et les corps artificiels de la photographie publicitaire}

5 Au Danemark, l'histoire de la photographie publicitaire d'un objet telle que nous la connaissons aujourd'hui remonte au début des années vingt. Avant cette époque, la publicité montrait le lieu de production de la marchandise, et parfois les ouvriers fabriquant le produit. Inspirés par la publicité américaine et par sa théorie, les publicitaires européens réalisèrent qu'il était plus profitable de présenter l'objet seul ou dans un décor artificiel. Parmi eux, Herman Bente, chef de la première agence de photographie publicitaire danoise, dénommée Jonals Co., possédait à la fois le matériel et la volonté nécessaires pour effectuer ce changement ${ }^{3}$.

Fig.1.

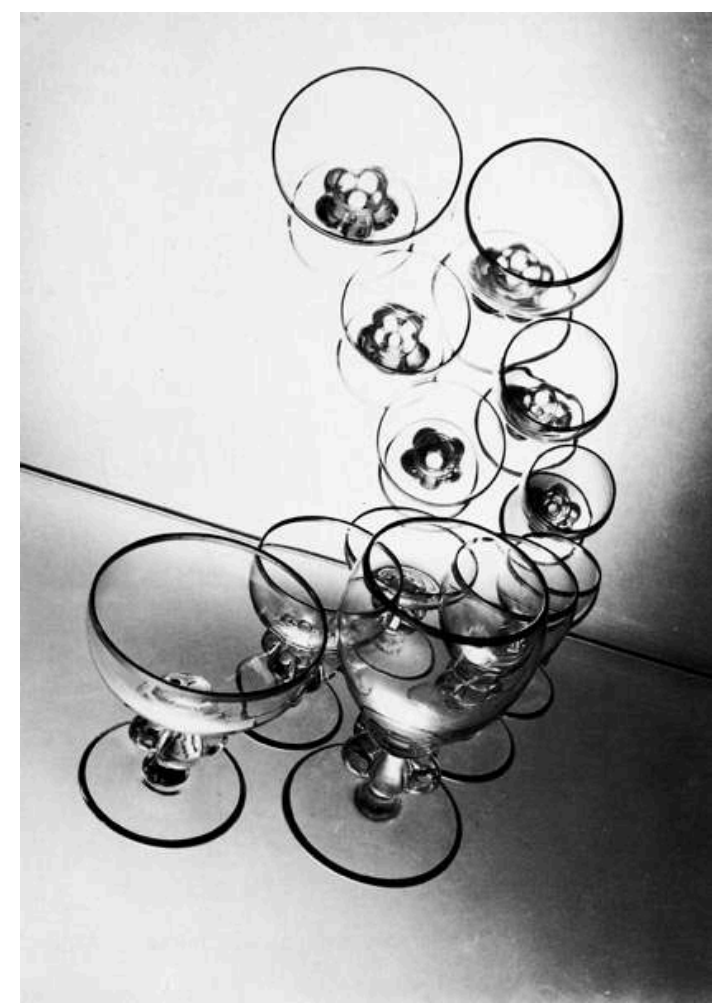

Jonals Co., (ca. 1933). TV-Arkiv og Research Center, Danmarks Radio.

Bente introduisit au Danemark la photographie publicitaire montrant le produit d'une façon détaillée. Comme ses contemporains, il insistait sur le fait que la photographie représente les objets d'une manière plus réaliste que la peinture ou le dessin. Les photographies de Jonals Co. manifestaient néanmoins aussi souvent un élément fantastique ou surréel. Celui-ci pouvait apparaître dans la structure même des objets, 
qui, éclairée et captée par les lentilles lucides et retenue sur le papier photographique hypersensible, se présentait de manière extrêmement distincte, ou dans leur mise en scène, comme dans l'image des verres, où les objets donnent l'impression d'avoir perdu leur pesanteur et de flotter dans l'air (fig. 1).

La photographie était ainsi capable de séparer la marchandise industrielle de ses circonstances de production - une séparation analogue à celle dont parle Karl Marx dans son célèbre texte, «Der Fetischcharacter der Ware und sein Geheimnis » de Das Kapital (1867) ${ }^{4}$. Mais c'est aussi la photographie publicitaire qui, et ce de la façon la plus accomplie, réussit à convertir la marchandise en fétiche ${ }^{5}$.

Le produit commercial, apparaissant comme un objet magique de la société de consommation, était aussi perçu comme un fétiche d'une façon plus contemporaine : à cette époque Sigmund Freud avait introduit la notion de fétiche en psychanalyse et, avec l'interprétation des symboles, elle constituait un nouveau cadre dans lequel l'animation de l'objet pouvait être comprise. Si l'on en croit la description du milieu publicitaire livrée par le romancier Niels Anesen, les photographes publicitaires avaient adopté l'interprétation sexuelle des objets proposée par Freud, et l'avaient incorporée directement dans leur travail. Bien avant que la bouteille de Coca Cola ne soit interprétée comme un phallus par la psychanalyse vulgaire, les photographes voyaient, selon Anesen, des formes sexuelles dans les objets. Dans le roman Skaf os et nyt ansigt (Fournissez-nous un nouveau visage) paru en 1943, on suit la jeune vendeuse Grete, qui fait son entrée dans les ateliers des photographes en tant que modèle de publicité. Elle pense à un photographe nommé Vallin, et s'exclame à son sujet : "En Seng var en Hun og en Divan en Han, det havde Vallin sagt, og han talte saa tit om Tingenes Væsen, det havde han sat sig ind $i$ for at faa Billeder og Annoncer til at virke $i$ Væsen og i Køn » («Un lit était une femelle et un divan était un mâle, c'est ce qu'avait dit Vallin, et il parlait souvent de la nature des objets, il s'était familiarisé avec les objets pour les faire vivre dans les images et dans les publicités selon leur essence et leur genre $"$, ma traduction) ${ }^{6}$. 
Fig.2.

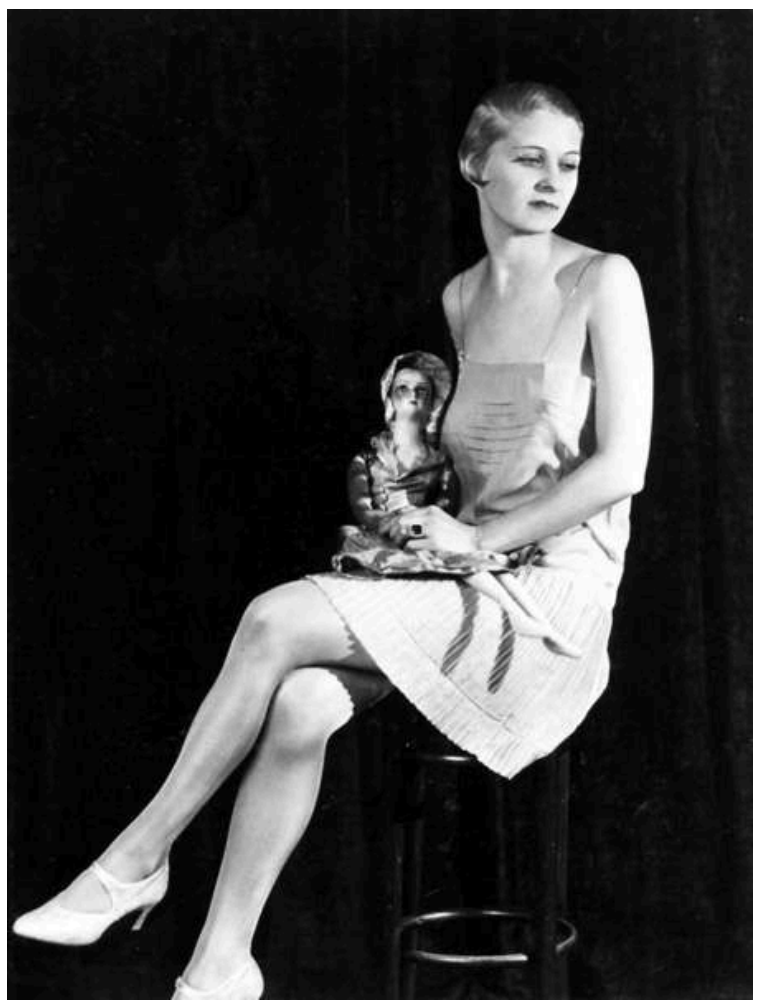

Jonals Co., 1928, Mode-Revy. E'nMagasin du Nords arkiv.

9 En ce qui concerne les «Grete » de cette époque, nous observons que, alors que le lit devient sexué et à cet effet un objet corporel, les femmes étaient quant à elles mises en scène comme des poupées ou des statues (fig. 2). Dans les photographies des journaux de mode, leurs corps rigides et le maquillage pâle les font ressembler à des poupées. La blancheur de leur peau, au-delà du maquillage, est rehaussée par la chimie noir et blanc de la photographie de l'époque. L'arrêt du mouvement que leur fait subir la photographie renforce encore le côté artificiel de leurs mimiques et leur donne irrémédiablement l'apparence d'automates.

\section{Les objets d'art vibrants et les corps décomposés}


Fig.3.

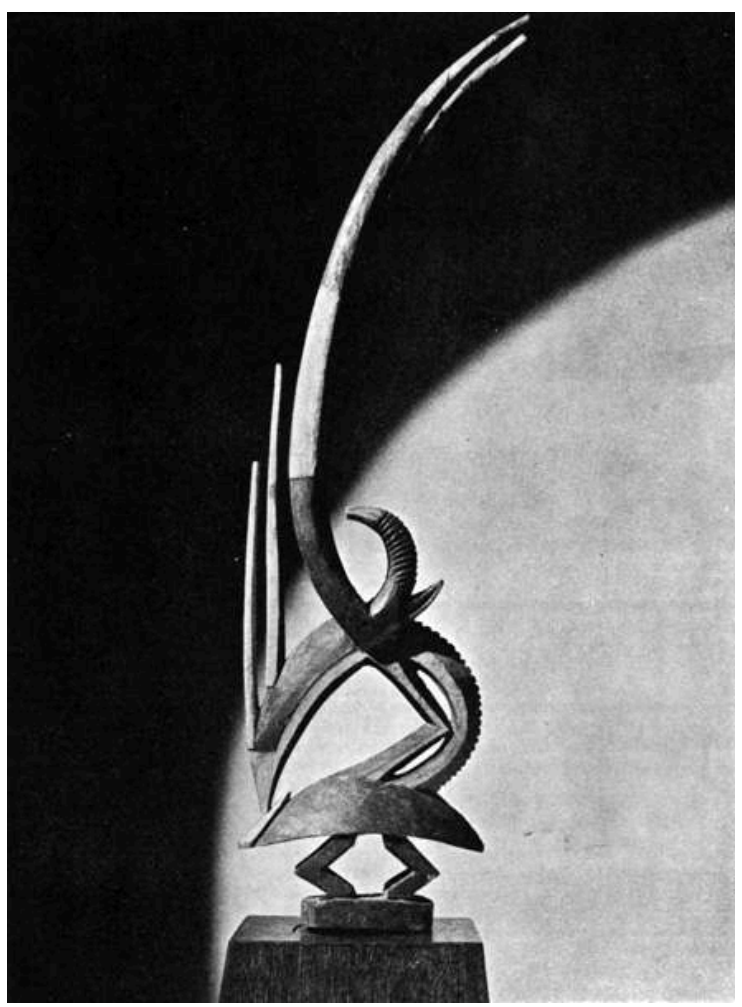

Vagn Guldbrandsen, Bambara. Dans Linien/ nr. 10, 1935.

10 L'analyse freudienne des symboles a inspiré les artistes danois, en particulier ceux qui se groupaient autour des deux revues Linien et Helhesten. On connaissait dans ces cercles les revues surréalistes françaises, notamment Minotaure dans laquelle le dadaïste et surréaliste Tristan Tzara déployait en 1933 sa théorie des formes sexuées dans la mode des chapeaux de l'époque ${ }^{7}$. L'article de Tzara « D'un certain Automatisme du Goût » eu une influence si profonde sur certains artistes danois, qu'ils écrivirent un article similaire dans Linien ${ }^{8}$. En ce qui concerne les illustrations, on ne peut pas comparer les images de l'article danois aux célèbres photographies de Man Ray montrant des chapeaux d'hommes et de femmes. Cette revue contient néanmoins un grand nombre de photographies spectaculaires montrant des objets dramatiques, dues, le plus souvent, aux photographes publicitaires mentionnés plus haut. Les objets d'art y sont photographiés de manière à souligner leur force exceptionnelle, de caractère à la fois anthropologique et psychanalytique. Le photographe publicitaire Vagn Guldbrandsen n'avait d'ailleurs pas seulement fait des photos de «marchandises fétiches ", mais aussi de fétiches africains de la collection du juriste Carl Kjersmeier une collection à laquelle les artistes danois portaient un vif intérêt (fig. 3) ${ }^{9}$. 
Fig.4.

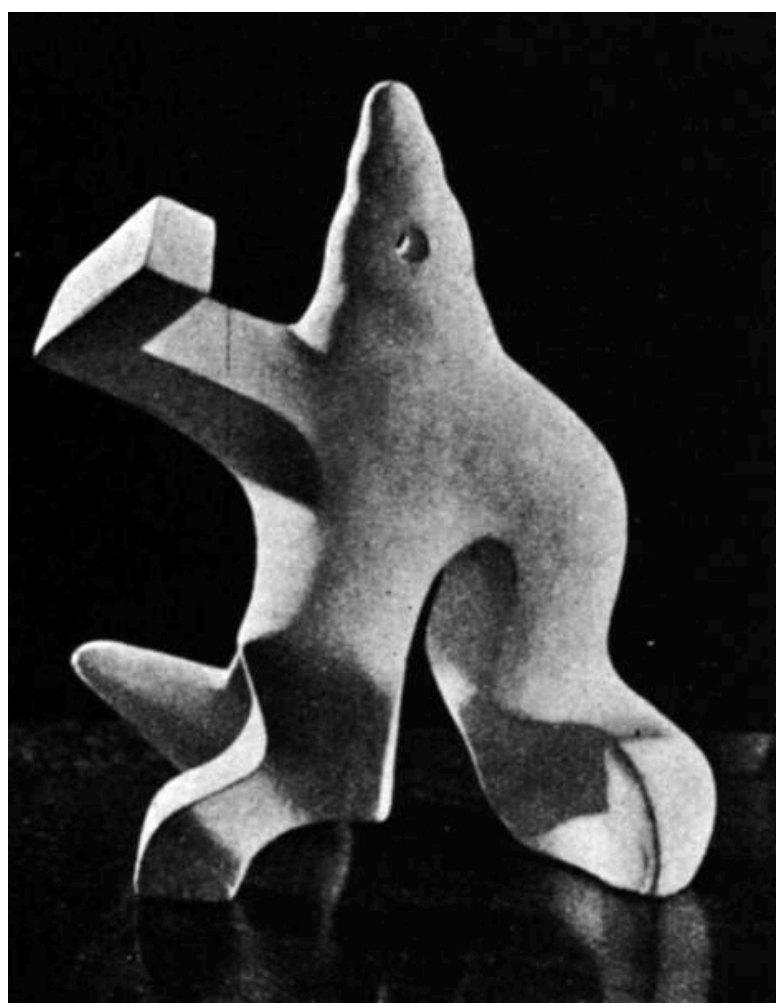

Jonals Co., Spadserende form (1933) de Ejler Bille. Dans Linien Julefluen, 1935.

11 Le style dramatique de ses photos a pour beaucoup contribué à rendre l'objet exotique et mystique, tout comme celui des photographies des sculptures de l'artiste Ejler Bille prises par Jonals Co. La puissante lumière y accentue la forme des objets, qui semble à la fois se cacher et se révéler. Bille définissait lui-même « le plastique » comme une « $\mathrm{i}$ spændingsforholdene bunden kraft. En ro, der er trængt ud til overfladen, men således, at man mærker nerven bagved, den bundne uro, voldsomheden, den inderste dirrende streng». («force contenue dans les relations de tension. Un calme qui paraît à la surface, mais d'une telle manière que, en dessous, l'on ressent la force retenue, l'agitation immobilisée, la violence, le frisson intime ", ma traduction) ${ }^{10}$. En regardant ces photographies, on a bien la sensation que l'acte photographique renforce cette impression d'énergie comprimée dans l'objet. La métamorphose mise en œuvre par la sculpture Spadserende Form (1933) (Forme se promenant, ma traduction) par exemple, semble réelle; elle apparaît comme une véritable transformation dont le processus aurait simplement été arrêté par l'obturateur de l'appareil photographique (fig. 4). 
Fig.5.

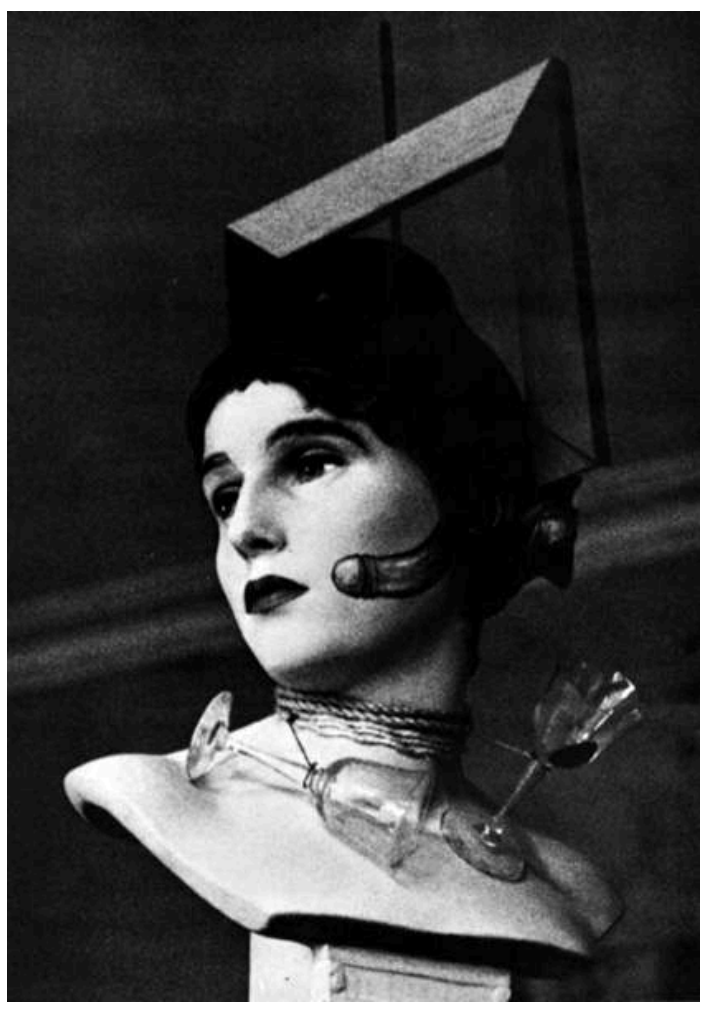

Wilhelm Freddie, Sex-paralysappeal (1936). Dans Jaguer : Wilhelm Freddie, Svend Hansens Forlag, 1969.

Il existe, dans les revues surréalistes danoises, un grand nombre d'autres exemples de photographies où les objets paraissent animés. Ainsi, certains clichés d'objets trouvés avec des marques d'usures racontent en même temps l'histoire de la vie du possesseur et celle de l'objet. Dans les images d'objets fétiches de l'artiste Freddie, les chaussures ou les gants empiètent sur des statues. L'œuvre la plus connue parmi celles-ci est sans doute Sex-paralysappeal (1936), où Freddie exploite la notion du désir entendue comme l'envie d'être proche de quelque chose ou de quelqu'un d'éloigné. Le spectateur reste à l'écart des choses, et la photographie de l'œuvre semble intensifier la sensation de proximité et en même temps d'éloignement de l'objet (fig. 5). En ce qui concerne Sexparalysappeal il faut ajouter que l'œuvre était uniquement accessible sous forme de photographie. L'objet, considéré comme pornographique, fut en effet confisqué par la police immédiatement après sa création. 
Fig.6.

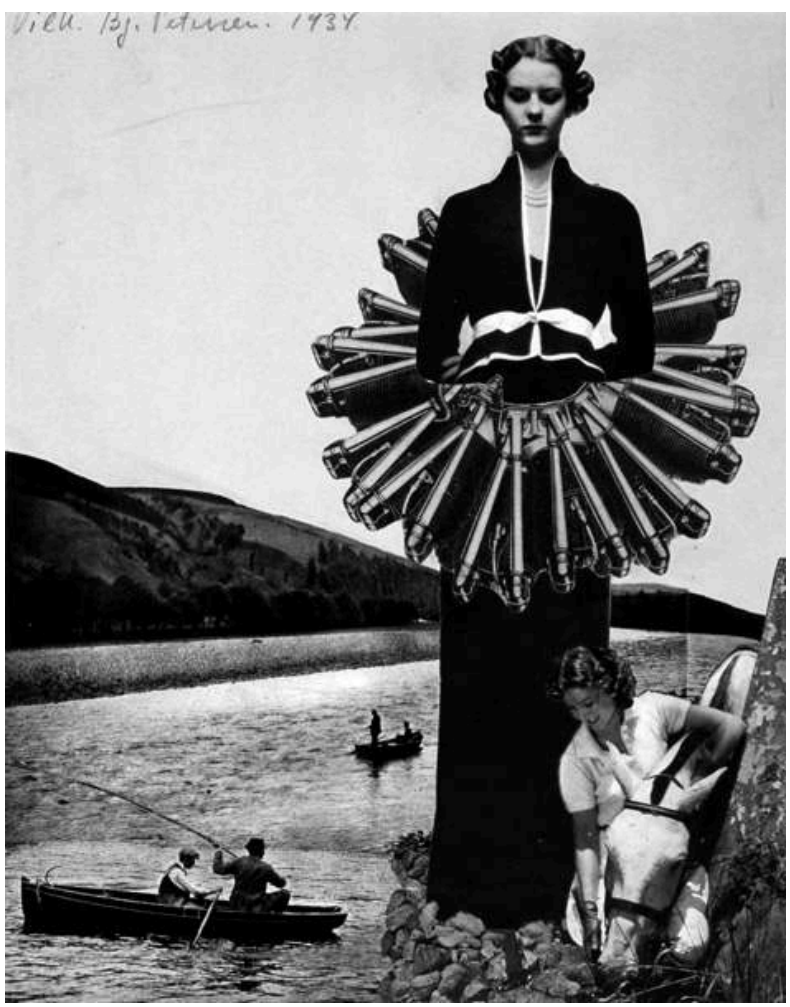

Vilhelm Bjerke Petersen, Montage, 1934. Silkeborg Kunstmuseum, Lars Bay

Si, dans les œuvres des surréalistes danois, l'objet est animé d'une manière anthropologique et psychanalytique, le corps subit, lui, une transformation extraordinaire dans leurs photomontages. Il est découpé, assemblé à des objets, créant ainsi des êtres à l'anatomie fantastique, mi corps, mi objet, sorte de chimère, à la fois organique et anorganique (fig. 6).

\section{Les objets hantés des occultistes et les médiums}

Dans une interview de 1935, Freddie affirmait que, selon lui, les objets avaient « une profondeur magique», qui s'exprimait dans leurs manières d'«attirer et de repousser $»^{11}$. Comme son maître Salvador Dali, il se servait souvent d'expressions comme "fantôme ", «spectral », " magique » etc. Il se rapprochait ainsi de l'univers occulte, où l'idée d'une vie propre des objets n'était pas seulement une représentation métaphorique, mais une réalité. Dans le monde occultiste, il y avait une division entre d'un côté les spirites, qui croyaient que les objets étaient animés par les esprits, et de l'autre les psychiques, qui affirmaient plutôt l'existence de forces physiques inconnues. Alfred Nielsen, de la Mission Spirite, soutenait par exemple l'hypothèse que les choses étaient hantées par les âmes des morts, tandis que le professeur de chimie Christian Winther cherchait des explications «rationnelles» dans les régions obscures des sciences naturelles. Toutefois, les psychiques et les spirites s'accordaient la plupart du temps sur l'idée que l'appareil photographique ne faisait que documenter la réalité des objets animés. Cette croyance a agacé les sceptiques, par exemple le professeur de psychologie Alfred Lehmann, qui avait avancé dans son œuvre Overtro og Trolddom 
(Superstition et Magie), que les images étaient le résultat d'une mise en scène de grande envergure ${ }^{12}$.

Les photographies danoises dites surnaturelles révèlent un univers où la frontière entre l'animé et l'inanimé semble dépassée. On «documentait» des pendules et des tables en donnant l'impression qu'elles bougeaient et flottaient. Les médiums étaient photographiés pendant qu'ils "matérialisaient» une "matière inconnue». Les croyants nommaient cette dernière "ectoplasme ». Les sceptiques répliquaient que c'étaient des morceaux de tissu - ou même des photographies - parfois cachés par le médium, parfois régurgités par lui.

Fig.7.

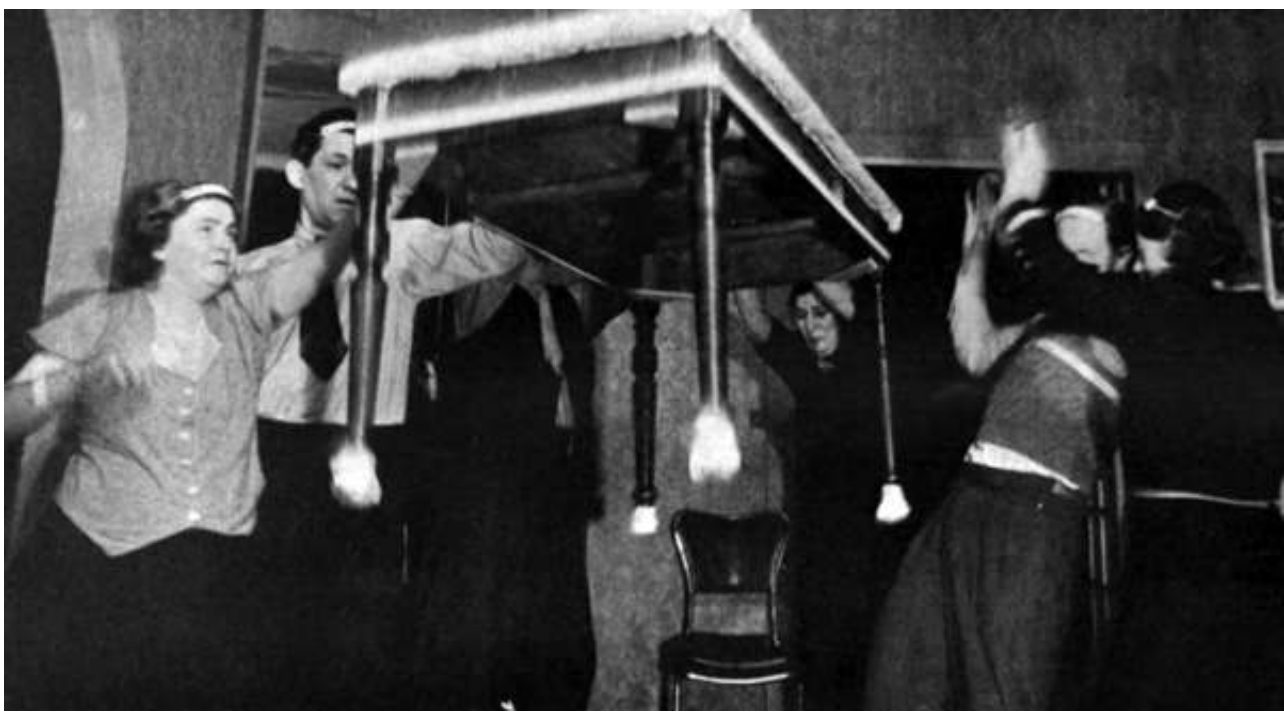

Sven Turck, Jeg var dus med.

Plusieurs photographes travaillaient en même temps dans différents domaines. Comme on l'a vu, Vagn Guldbrandsen était à la fois photographe publicitaire et photographe artistique. Sven Türck s'est également révélé comme photographe transdisciplinaire, mais pour lui, la publicité allait de pair avec l'occultisme. Dans la journée Türck travaillait dans son atelier publicitaire, où il photographiait des marchandises avec "smæld og kraft» ("claquement et force») selon ses propres mots ${ }^{13}$. La nuit, il organisait des séances occultes pendant lesquelles il «documentait» des tables flottantes et autres phénomènes dits occultes (fig. 7) ${ }^{14}$.

Si l'on revient au texte "Le caractère fétiche de la marchandise et son secret », Marx a justement choisi la table comme exemple d'une vie propre de la marchandise lorsqu'elle s'est détachée de son mode de production. Marx se sert d'une métaphore expressive quand il écrit que la table se dresse sur sa tête de bois et qu'elle se livre à des caprices bizarres ${ }^{15}$. À ces propos, Marx ajoute les tables tournantes des spirites, mais pour lui la table-marchandise diffère essentiellement de la table spirite. Dans le cas de Türck, au contraire, il y a, au moins dans une perspective biographique, une cohésion entre la table tournante et la table commerciale, car elles étaient toutes deux animées par le photographe. Cela évoque les thèses d'un autre marxiste, Theodor W. Adorno, selon lequel l'occultisme était une maladie capitaliste générale. Dans l'article « Thesen gegen den Okkultismus", il établit un lien entre l'aliénation de la société de consommation et les notions d'objets hantés des occultistes. Si l'on suit le 
raisonnement d'Adorno, il est évident que Türck pourrait être diagnostiqué comme un cas grave. La question est donc de savoir dans quelle mesure Türck est conscient d'être contaminé. Est-il un homme d'affaires qui, de manière calculatrice, exploite le désir d'explorer l'inconnu? Ou bien est-il persuadé de l'existence des phénomènes surnaturels et, dans une limite restreinte, la victime d'une illusion ? Quelle que soit la réponse à ces questions fondamentales, on constate que la photographie était un instrument indispensable à l'animation de l'objet occulte ainsi qu'à l'animation du produit commercial.

Fig.8.

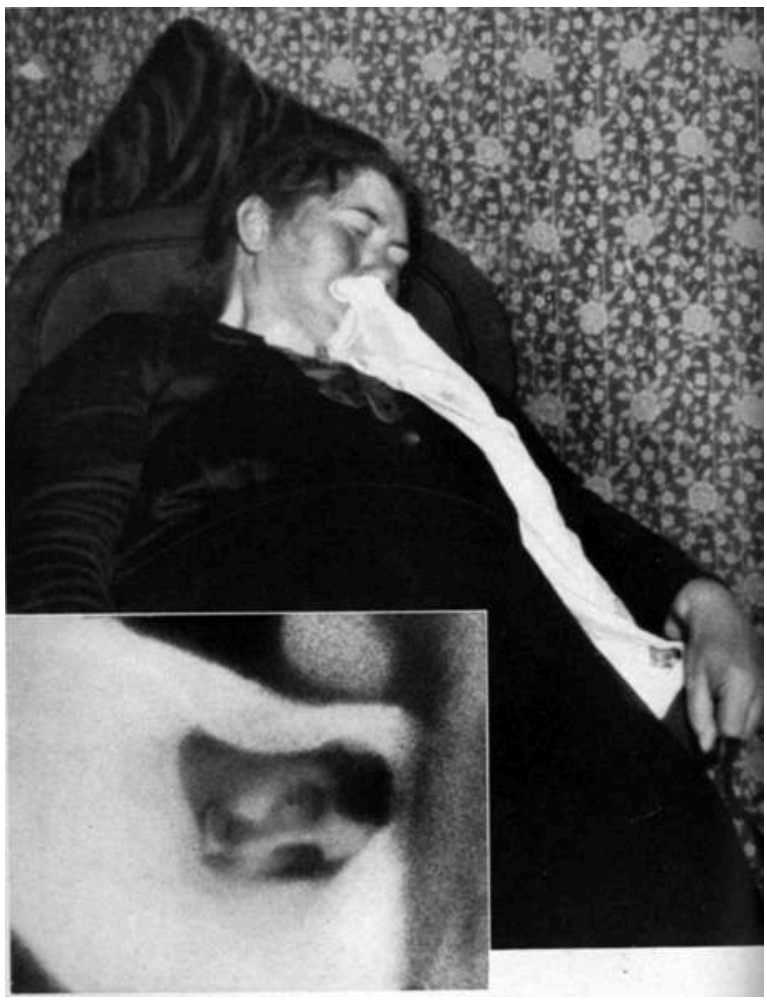

Johanne Stoltz. Dans Sven Turck, Jeg var dus med

Quant à l'autre partie de l'inquiétante étrangeté, c'est-à-dire le corps qui semble être mort, les médiums étaient, bien entendu, perçus comme des êtres mécaniques (fig. 8). Lorsqu'on les documentait, c'était pour démontrer qu'il était possible qu'ils soient dirigés par des âmes ou des forces inconnues.

19 L'historienne de la photographie peut se limiter à étudier la manière dont la photographie soutient la notion de médium comme endormi ou en transe, mais elle peut aussi élargir la perspective. Sans doute y a-t-il des échanges entre le processus photographique et la métaphysique occulte. Tous deux sont des systèmes mécaniques et matérialistes. Les séances sont en partie comprises comme des chambres noires dans lesquelles les médiums, les appareils, captent les âmes-images. 


\section{L'histoire culturelle et la photographie}

domaines, au premier abord, très éloignés les uns des autres. Il est possible d'identifier une inquiétante étrangeté dans l'idée d'objets animés et de corps morts ou mécaniques, présente dans la publicité, dans l'art et dans l'occultisme, et d'étudier comment ces idées sont liées entre elles. Cette inquiétante étrangeté s'exprime également dans la photographie. Rappelons que le monde à cette époque, comme l'a écrit en 1928 le théoricien allemand Siegfried Kracauer, a " un visage photographique ", c'est-à-dire que la vision du monde devient de plus en plus influencée par les images photographiques ${ }^{16}$. C'est pourquoi on considère l'inquiétante étrangeté comme un élément photographique aussi bien que comme un élément philosophique et sociologique. On prête surtout attention à la manière dont l'image photographique influence la notion d'objet et de corps. Les agents photographiques, c'est-à-dire l'illumination, la mise en scène, l'arrêt, l'absence etc., instaurent un changement des notions d'animé et d'inanimé. C'est grâce à la photographie que l'objet semble échapper à la pesanteur, devenir lumineux, et que les corps apparaissent comme pétrifiés ou mécaniques. De cette manière, l'image photographique entre autant dans différents systèmes de signification culturels qu'elle les forme. Elle fait partie de la notion d'apesanteur ou de lumière dans la culture commerciale, dans l'art, dans la pseudo-science et dans la religion.

La photographie influence la notion du monde mais aussi la manière dont on le perçoit. Vus à travers la photographie, c'est non seulement la nature de l'objet et du corps qui est différente, mais aussi la relation entre le motif et le spectateur. La photographie laisse le spectateur regarder en profondeur le motif de l'image - mais toujours à distance de l'objet même. Cela implique que l'expérience d'attraction et de répulsion change aussi, ce qui est exploité dans tous les domaines mentionnés. L'étude de la notion d'inquiétante étrangeté dans la photographie danoise de l'entre-deux-guerres est un voyage parmi les notions d'objets et de corps dans différents domaines, ainsi qu'une excursion qui éclaire sous un jour nouveau le médium photographique.

\section{NOTES}

1. Sigmund Freud, «Das Unheimliche» (1919), Gesammelte Werke . Vol. XII. London, Frankfurt am Main, Imago Publishing Co., S. Fischer Verlag, 1947.

2. Ernst Jentsch, «Zur Psychologie des Unheimlichen I-II», Psychiatrisch-Neurologische Wochenschrift no. 22-23, 1906, pp.195-198, pp. 203-205.

3. Cf. Mette Kia Krabbe Meyer, «Fotografi uden kruseduller», Jonals Co. og det moderne fotografis gennembrud i Danmark, København, Gl. Holtegaard, 2000, pp. 7-55. Et «Et direkte udsnit af virkeligheden? 1920'ernes rene fotografi», Dansk fotografihistorie, Ed. Mette Sandbye, København, Gyldendal, 2004.

4. Karl Marx, «Der Fetischcharacter der Ware und sein Geheimnis» (1867), Ökonomische Schriften, Ed. Benedikt Kaufsky, Stuttgart, Cotta Verlag, 1962, pp. 46-63.

Images Re-vues, 4 | 2007 
5. Bien qu'il ait emprunté la notion de fétiche à l'anthropologie, où le mot est employé lorsqu'on parle d'idoles magiques, Marx n'analysait pas la marchandise comme un objet magique. Le mérite d'une telle analyse reviendra, bien plus tard, à Jean Baudrillard. Cf. Jean Baudrillard, La société de consommation, 1970, Paris, Éditions Denoël, 1991.

6. Niels Anesen, Skaf os et nyt Ansigt, København, Pantheon Forlag, 1943, p. 215.

7. Tristan Tzara, «D'un certain Automatisme du Goût», Minotaure, vol. 1, no. 3-4, 1933, pp. 81-84.

8. Don Juan: «er det sexuelle grundbegrebet for hattens æstetik? », Linien, vol. 1, no. 3, 1934, Esbjerg Kunstforening, 1984, p. 4.

9. Cf. Carl Kjersmeier, Centres de style de la sculpture nègre africaine, I-IV vols, Paris, København, Éditions Albert Morancé, Illums Bog-Afdeling, Fischers Forlag, 1935-1938. Negerskulptur . Alverdens Kunst, Ed. Jens Yde, København, Rosendahl \& Jørgensens Forlag, 1946.

10. Ejler Bille, «Alberto Giacometti», Helhesten, vol. 1, no. 3, 1941, p. 80-81.

11. Cité par Rolf Læssøe, Wilhelm Freddie, København, Søren Fogtdal, 1996, p. 64.

12. Alfred Lehmann, Overtro og Trolddom. Fra de ældste Tider til vore Dage, Vol. 1-4, København, J. Frimodts Forlag, 1893-1896.

13. Sven Türk, Kursus I fotografi. IV lekion, København, Teknik Kursus, Bogtrykkeriet Antikva A/ S, 1949-1950, p. 152.

14. En 1945 Türck a publié le livre Jeg var dus med Aanderne ( Je tutoyais les esprits ), un récit dramatique d'expériences avec des tables tournantes et des médiums se matérialisant, agrémenté d'éléments d'explication pseudo-scientifiques. Il est illustré avec des clichés de Türck et d'autres photographes danois. Sven Türck, Jeg var dus med Aanderne, København, Steen Hasselbalchs Forlag, 1945. Cf. Mette Kia Krabbe Meyer: «Mørkekammerets blændværk. Okkultisme og fotografi», Kritik 161, 2003, Le troisième oeil : La photographie et l'occulte, Clément Chéroux et al., Gallimard, 2004, p. 244-245.

15. Karl Marx, «Der Fetischcharacter der Ware und sein Geheimnis» (1867), Ökonomische Schriften, Ed. Benedikt Kaufsky, Stuttgart, Cotta Verlag, 1962, p. 46.

16. Siegfried Kracauer, «Die Photographie», Frankfurter Zeitung, 28/10, 1927, Das Ornament der Masse . Frankfurt, Suhrkamp, 1977, p. 34.

\section{RÉSUMÉS}

L'auteure étudie la manière dont la photographie danoise de l'entre-deux-guerres joue des objets représentés pour leur donner cette "inquiétante étrangeté " que Freud avait théorisée à l'époque. En comparant la photographie publicitaire, les montages surréalistes et la photographie spirite, l'analyse décrypte les mécanismes mis en place pour « animer » des objets inanimés ou au contraire pour pétrifier des corps vivants, et met ainsi en évidence des liens entre des domaines photographiques a priori très distincts.

\section{INDEX}

Index géographique : Danemark

Mots-clés : corps, occultisme, produit, psychanalyse, surréalisme, symbole

Index chronologique : XXe siècle

Thèmes : photographie 


\section{AUTEUR}

\section{METTE KIA KRABBE MEYER}

Mette Kia Krabbe Meyer est titulaire d'un PhD de l'Université de Copenhague (Institut d'Art et de Sciences Humaines) est actuellement conservatrice au Musée Steno (Musée Danois de l'Histoire de la Science). Elle a écrit notamment sur la photographie danoise des années vingt dans Dansk fotografihistorie, Mette Sandbye ed., København 2004.

SIMK@phys.au.dk 